\title{
Relação do Índice de Massa Corporal e Linfedema Secundário a tratamento cirúrgico do Câncer de Mama: estudo retrospectivo
}

\author{
Relationship of Body Mass Index and Secondary \\ Lymphedema to Surgical Treatment of Breast Cancer: \\ a retrospective study
}

\author{
Marcelo Antonini' \\ Gisela Rosa Franco Salerno ${ }^{2}$ (1) \\ Raquel dos Santos Moro 3 (1) \\ Maria Virginia de Oliveira e Oliveira ${ }^{4}$ \\ Odair Ferraro 5 \\ Reginaldo Guedes Coelho Lopes ${ }^{6}$ (1)

\begin{abstract}
1,3,4,6!nstituto de Assistência Médica ao Servidor Público Estadual de São Paulo (São Paulo). São Paulo, Brasil. drantonini@uol.com.br, raquel_smoro@hotmail.com,dra.mariavirginia@gmail.com, jarelu@uol.com.br

${ }^{2}$ Autora para correspondência. Universidade Presbiteriana Mackenzie (São Paulo). São Paulo, Brasil. gisela.franco@mackenzie.br
\end{abstract} \\ ${ }^{5}$ Hospital do Servidor Público Estadual. Instituto de Assistência Médica ao Servidor Público Estadual de São Paulo (São Paulo). São Paulo, Brasil.
} odairferraro@hotmail.com

RESUMO | INTRODUÇÃO: O câncer de mama é a neoplasia mais incidente na população feminina brasileira, sendo o linfedema uma de suas principais complicações cirúrgicas. OBJETIVO: Verificar a relação do índice de massa corpóreo e o desenvolvimento do linfedema no pós-operatório de câncer de mama, sua relação com o tipo de tratamento e ao tempo pós-cirúrgico. MATERIAIS E MÉTODOS: Foram levantados prontuários de 59 mulheres no período de maio de 2008 a fevereiro de 2009 utilizando dados da anamnese; exame físico; perimetria, volume estimado; relatos, sintomas e dados em prontuário. A análise foi feita por meio de médias e desvios padrões para as variáveis quantitativas e qualitativas (absoluta e relativa) e para verificação dos fatores de risco foi utilizado o Teste t de Student e Teste Qui-quadrado de Pearson com nível de significância de 5\%. RESULTADOS: A frequência de linfedema foi de $47,5 \%$, sendo que $40,7 \%$ eram obesas. Observou-se diferença significativa entre os grupos de linfedema e IMC $(p=0,002)$. Nota-se ainda diferença significativa entre linfedema no grupo sem radioterapia de axila $(p=0,003)$ e associação entre o tempo pós-cirúrgico e linfedema $(p=0,006)$, sendo maior após 6 meses de cirurgia. CONCLUSÃO: Existe correlação entre obesidade e desenvolvimento do linfedema. Os tipos de tratamento parecem não ter influenciado o seu desenvolvimento e quanto mais tempo decorrido pós- cirurgia, maior o risco do seu aparecimento.

PALAVRAS-CHAVE: Neoplasias da mama. Linfedema Relacionado a Câncer de Mama. Obesidade. Procedimentos Cirúrgicos Eletivos.
ABSTRACT | INTRODUCTION: Breast cancer is the most common neoplasm in the Brazilian female population, with lymphedema being one of its main surgical complications. OBJECTIVE: To verify the relationship between the body mass index and the development of lymphedema in the postoperative period of breast cancer, its relationship with the type of treatment received and the time elapsed from the surgery. MATERIALS AND METHODS: Medical records of 59 women were collected from May 2008 to February 2009 using data from anamnesis, physical exam; perimetry, estimated volume; reports, symptoms, and notes in medical records. The analysis was performed using means and standard deviations for the quantitative and qualitative variables (absolute and relative) and to verify the risk factors used, the Student's t-test and Pearson's chi-square test with a 5\% significance level (0.05). RESULTS: The frequency of lymphedema was $47.5 \%$, with $40.7 \%$ being obese. There was a significant difference between the groups of lymphedema and BMI $(p=0.002)$. There is also a significant difference between lymphedema in the group without axillary radiotherapy $(p=0.003)$ and an association between post-surgical time and lymphedema $(p=0.006)$, being greater after six months of surgery. CONCLUSION: There is a correlation between obesity and the development of lymphedema. The types of treatment do not seem to have influenced their development, and the longer the surgery has elapsed, the greater the risk of their appearance.

KEYWORDS: Breast Neoplasms. Breast Cancer Lymphedema. Obesity. Elective Surgical Procedures. 


\section{Introdução}

A neoplasia maligna da mama é o segundo tipo mais frequente de câncer no mundo e o mais comum entre as mulheres. A cada ano, $25 \%$ dos novos casos de câncer em mulheres são mamários. No Brasil foram estimados 66.280 casos novos de câncer de mama para o ano de 2021, e o número de mortes foi de 16.724 no ano de 2017. Estatísticas indicam aumento da incidência tanto em países desenvolvidos quanto aqueles em desenvolvimento. ${ }^{1}$

O rastreamento mamográfico permite a detecção do câncer mamário mais precoce, favorecendo condutas mais efetivas e menos agressivas garantindo o aumento da sobrevida das mulheres.? Por outro lado, o aumento na sobrevida leva também ao aumento na incidência do linfedema, que é uma doença crônica e de difícil tratamento, ocasionando desordens funcionais, psicológicas e sociais para as portadoras de câncer de mama. ${ }^{3}$

Das complicações relacionadas ao tratamento cirúrgico do carcinoma mamário, o linfedema tem implicações relevantes, pois além de sua fisiopatologia ainda não ser totalmente esclarecida, apresenta prevalência de $12 \%$ a $30 \%$, variando consideravelmente de acordo com o tratamento proposto. O início do linfedema pode ser precoce, isto é, logo no pós-operatório, ou tardio, surgindo anos após a cirurgia. ${ }^{4}$

Segundo o Consenso da Sociedade Internacional de Linfologia ${ }^{5}$, o linfedema é uma manifestação clínica de insuficiência do sistema linfático, com consequente desordem no transporte de linfa. Ampliando para o câncer de mama, o linfedema é uma complicação de morbidade alta, que afeta diretamente a qualidade de vida e perfil psicológico da paciente. $\underline{6}$ Sabe-se que os principais fatores de risco para o desenvolvimento do linfedema são a radioterapia, infecção, obesidade e número de linfonodos retirados. Já o tamanho do tumor e sua localização não foram associados a maior risco de linfedema.

Todavia, seu diagnóstico pode ser obtido por meio de sintomas e sinais clínicos apresentados pelas pacientes. Dentre os sintomas relatados, a dor, parestesias, diminuição de função e edema são os mais frequentes. Já entre os sinais clínicos estão as alterações na perimetria, volumetria e ultrassonografia; entretanto, os exames complementares só são indicados quando existe dúvida no diagnóstico. $\frac{3}{}$
Ademais o linfedema pode ser classificado como, leve (inferior a $3 \mathrm{~cm}$ ), moderado $(3 \mathrm{a} 5 \mathrm{~cm}$ ) e severo (superior a $5 \mathrm{~cm}$ ), sendo que quanto maior a perimetria do membro, mais aderências, menos funcionalidade do membro e maiores dificuldades de tratamento, necessitando, portanto, da prevenção como melhor estratégia terapêutica quando os fatores de risco estão presentes..,$\underline{7}$ Dentre os fatores de risco modificáveis para controle do linfedema de membros superiores está a obesidade, ou seja, o controle de peso tornou-se uma recomendação nos consensos e Guidelines atuais, sendo ressaltado tanto no período pré e pós-cirúrgicos.

Enfim, o linfedema é uma das complicações advindas do tratamento do câncer de mama que traz consigo reações físico-funcionais e psicológicos que poderão interferir diretamente na qualidade de vida dessa população. Medidas de identificação desses fatores de risco para seu desencadeamento poderá nos direcionar aos protocolos específicos de prevenção. Sendo assim, tornou-se relevante avaliar a relação do índice de massa corpóreo e o desenvolvimento do linfedema no pós-operatório de câncer de mama, sua relação com o tipo de tratamento recebido e o tempo pós-cirúrgico.

\section{Material e métodos}

Foi realizado um estudo retrospectivo no período de maio de 2008 a fevereiro de 2009 com 59 prontuários de mulheres submetidas ao tratamento cirúrgico de neoplasia maligna mamária de um Hospital público na Cidade de São Paulo. Foram incluídas para essa investigação prontuários de mulheres que realizaram cirurgia mamária por neoplasia maligna, excluindo os que relatavam cirurgia mamária bilateral, tempo da cirurgia menor que três meses, presença de cirurgia axilar e linfedema crônico prévio.

Toda a tabulação de dados foi realizado por um mesmo investigador, considerando as seguintes variáveis; idade, índice de massa corpóreo (IMC), estadiamento clínico pré-cirúrgico, tipo da cirurgia, tempo decorrido até o momento, abordagem axilar considerando nível de esvaziamento além do número de linfonodos acometidos verificados no anatomopatológico, adjuvância com radioterapia locorregional e axilar, quimioterapia, hormonioterapia, presença de complicações como seroma, hematoma e infecções da ferida operatória. 
E para identificação do linfedema foram transcritos os valores da perimetria apresentada nos prontuários na parte de exame físico, sendo identificado como linfedema a apresentação da diferença de $2 \mathrm{~cm}$ na perimetria comparado ao membro contralateral. As marcações perimétricas foram realizadas em 3 pontos específicos do braço, 3 pontos específicos do antebraço, punho e mãos padronizados pela equipe do hospital. As avaliações eram sempre realizadas pela mesma pessoa para evitar erros nas marcações.

Os dados foram analisados e apresentados de forma descritiva por meio de médias e desvios padrões para as variáveis quantitativas e para as variáveis qualitativas frequências absolutas (N) e relativas (\%). As variáveis quantitativas, idade e IMC, apresentaram normalidade verificada pelo Teste de Kolmogorov-Smirnov (KS), para tanto, utilizamos Teste Qui-quadrado de Pearson, sendo considerado um nível de significância de $5 \%(\alpha=0,05)$ os testes teve conclusão sob hipótese bicaudal.

\section{Resultados}

Dos 59 prontuários analisados, foi encontrado idade média de $60 \pm 3,45$ anos, com IMC médio de $28,86 \pm 1,25 \mathrm{Kg} /$ $\mathrm{m} 2$, sendo que 40\%, 23 mulheres apresentavam IMC acima de $30 \mathrm{Kg} / \mathrm{m} 2$, ou seja, quadro de obesidade, a frequência de linfedema foi de $47 \%$ (28 mulheres).

A cirurgia conservadora foi realizada em $54 \%$ (33) mulheres, enquanto a cirurgia radical foi realizada em $45,8 \%$ (26) mulheres. Do ponto de vista do tratamento axilar 32,2\% (19) mulheres realizaram biópsia do linfonodo sentinela (BLS) e 67,8\% (40) pacientes realizaram linfadenectomia axilar, todas as pacientes que foram encaminhadas para linfadenectomia axilar apresentavam linfonodos comprometidos. Além disso, a complementação terapêutica com radioterapia (RT) mamária aconteceu em 66,1\% (39) mulheres.

Quanto à existência de comorbidades 66,1\% (39) mulheres apresentavam alguma doença crônica, sendo a mais prevalente em 56\% (33) mulheres a hipertensão arterial sistêmica (HAS) e 10\% (6) mulheres apresentavam diabetes mellitus (DM). Em relação às complicações apresentadas no pós-operatório, independentemente do tipo cirurgia, 76,3\% (45) mulheres apresentaram algum tipo de sintomatologia e 23,7\% (14) não apresentaram nenhuma intercorrência. Entre as complicações citadas, a infecção foi identificada em 95\% (43) delas, 90\% (40) apresentaram radiodermite, 95\% (43) apresentaram erisipela e seromas em 95\% (43) delas (Tabela 1).

Tabela 1. Características sociodemográficas, antropométrica e clínicas em 59 mulheres submetidas ao tratamento cirúrgico de neoplasia maligna mamária $2008-2009$

\begin{tabular}{|c|c|c|}
\hline Idade média & \multicolumn{2}{|c|}{$60 \pm 3,45$ anos } \\
\hline \multirow[t]{2}{*}{ IMC médio } & \multicolumn{2}{|c|}{$28,86 \pm 1,25 \mathrm{Kg} / \mathrm{m}^{2}$} \\
\hline & (N) $\%$ & (N) \% \\
\hline \multirow[t]{2}{*}{ IMC } & (23) $40 \%$ acima de $30 \mathrm{Kg} / \mathrm{m}^{2}$ & (36) $60 \%$ abaixo de $30 \mathrm{Kg} / \mathrm{m}^{2}$ \\
\hline & $(\mathrm{N}) \%$ realizaram & (N)\% não realizaram \\
\hline Biópsia do LS & (19) $32,2 \%$ & (40) $67,8 \%$ \\
\hline Linfadenectomia axilar & (40) $67,8 \%$ & (19) $32,2 \%$ \\
\hline \multirow[t]{2}{*}{ RT mamária } & (39) $66,1 \%$ & (20) $43,9 \%$ \\
\hline & (N)\% Presente & (N)\% Ausente \\
\hline Linfedema & (28) $47 \%$ & (31) $53 \%$ \\
\hline \multirow{3}{*}{ Comorbidades } & (39) $66,1 \%$ & \multirow{3}{*}{ (20) $43,9 \%$} \\
\hline & (33) $56 \%$ HAS & \\
\hline & (6) $10 \% \mathrm{DM}$ & \\
\hline Complicações no PO & $(45) 76,3 \%$ & \multirow{5}{*}{ (14) $23,7 \%$} \\
\hline Infecção & (43) $95 \%$ & \\
\hline Radiodermite & (40) $90 \%$ & \\
\hline Erisipela & (43) $95 \%$ & \\
\hline Seromas & (43) $95 \%$ & \\
\hline
\end{tabular}

IMC: Índice de massa corporal, LS: Linfonodo Sentinela, RT: Radioterapia, HAS: Hipertensão arterial Sistêmica, DM: Diabete Mellitus, PO: pós-operatório. 
A idade, IMC, características clínicas e cirúrgicas em relação ao linfedema e a relação ao linfedema estão descritas na Tabela 2. Quando verificado as diferenças entre as medidas das pacientes com e sem linfedema apresentaram significância estatística para o IMC com medidas maiores para o grupo com linfedema, média/desvio padrão de $31,3 \pm 5,8 \mathrm{Kg} / \mathrm{m} 2$, enquanto o grupo sem linfedema apresenta média/desvio padrão de $26,63 \pm 6,2 \mathrm{Kg} / \mathrm{m} 2$, com $p=0,002$.

Tabela 2. Associação entre a idade, IMC, características clínicas e cirúrgicas em relação ao linfedema em 59 mulheres submetidas ao tratamento cirúrgico de neoplasia maligna mamária 2008-2009

\begin{tabular}{|c|c|c|c|c|c|c|c|c|}
\hline & & \multicolumn{4}{|c|}{ Presença de Linfedema } & \multirow[b]{3}{*}{ Valor de $p$} & \multirow{2}{*}{\multicolumn{2}{|c|}{ Total }} \\
\hline & & \multicolumn{2}{|c|}{ Não } & \multicolumn{2}{|c|}{ Sim } & & & \\
\hline & & $\mathbf{N}$ & $\%$ & $\mathbf{N}$ & $\%$ & & $\mathbf{N}$ & $\%$ \\
\hline \multirow{2}{*}{ Idade } & Não jovem & 28 & 90,3 & 26 & 92,9 & $\geq 0,05$ & 54 & 91,5 \\
\hline & Jovem & 3 & 9,7 & 2 & 7,1 & $\geq 0,05$ & 5 & 8,5 \\
\hline \multirow{3}{*}{ IMC } & Normal & 15 & 48,4 & 2 & 7,1 & 0,002 & 17 & 28,8 \\
\hline & Sobrepeso & 7 & 22,6 & 11 & 39,3 & 0,002 & 18 & 30,5 \\
\hline & Obeso & 9 & 29,0 & 15 & 53,6 & 0,002 & 24 & 40,7 \\
\hline \multirow{3}{*}{ TNM } & 1 & 16 & 53,3 & 8 & 28,6 & $\geq 0,05$ & 24 & 41,4 \\
\hline & II & 8 & 26,7 & 15 & 53,6 & $\geq 0,05$ & 23 & 39,7 \\
\hline & III & 6 & 20,0 & 5 & 17,9 & $\geq 0,05$ & 11 & 19,0 \\
\hline \multirow{2}{*}{ Cirurgia } & Conservadora & 16 & 53,3 & 16 & 57,1 & $\geq 0,05$ & 32 & 55,2 \\
\hline & Radical & 14 & 46,7 & 12 & 42,9 & $\geq 0,05$ & 26 & 44,8 \\
\hline \multirow{2}{*}{$\begin{array}{c}\text { Axila } \\
\text { positiva }\end{array}$} & Não & 22 & 71,0 & 17 & 60,7 & $\geq 0,05$ & 39 & 66,1 \\
\hline & Sim & 9 & 29,0 & 11 & 39,3 & $\geq 0,05$ & 20 & 33,9 \\
\hline
\end{tabular}

IMC: índice de Massa Corpóreo. TNM: Classificação do American Joint Committee on Cancer (AJCC) e União Internacional de Controle do Câncer (UICC). Onde a letra T: Tumor primário, N: câncer disseminou para linfonodos próximos, M: Metástase: câncer disseminou para partes distantes do corpo. Variando sua classificação de I a IV. Informar o ponto de corte da idade, para a classificação utilizada em jovem e não jovem. Teste Qui-quadrado de Pearson.

Considerando a Tabela 3, que mostra a descrição dos tratamentos para o desenvolvimento do linfedema, observa-se que $67,9 \%$ (19) mulheres que receberam a RT na mama, desenvolveram o linfedema, enquanto 10,7\% (3) das que receberam RT na axila, desenvolveram o linfedema. Em relação a QT, 28,6\% (8) mulheres que receberam a quimioterapia, apresentaram linfedema, comparada a 35,5\% (11) que não desenvolveram tal alteração. Em relação a mulheres que receberam a HT, 29,5\% (14) desenvolveram linfedema comparada a 32,5\% (10) que não desenvolveram. Quando associado o tipo de tratamento recebido e o desenvolvimento do linfedema, houve diferença significantes estatisticamente somente comparando a não execução da RT na axila e o desenvolvimento do linfedema $(p=0,003)$.

Tabela 3. Associação entre as frequências de tratamentos em relação ao linfedema em 59 mulheres submetidas ao tratamento cirúrgico de neoplasia maligna mamária 2008-2009

\begin{tabular}{|c|c|c|c|c|c|c|c|c|}
\hline & & \multicolumn{4}{|c|}{ Presença de Linfedema } & \multirow[b]{3}{*}{$\begin{array}{c}\text { Valor de } \\
\mathbf{p}\end{array}$} & \multirow{2}{*}{\multicolumn{2}{|c|}{ Total }} \\
\hline & & \multicolumn{2}{|c|}{ Não } & \multicolumn{2}{|c|}{ Sim } & & & \\
\hline & & $\mathbf{N}$ & $\%$ & $\mathbf{N}$ & $\%$ & & $\mathbf{N}$ & $\%$ \\
\hline \multirow{2}{*}{$\begin{array}{c}\text { RT. } \\
\text { Mama }\end{array}$} & Não & 11 & 35,5 & 9 & 32,1 & $\geq 0,05$ & 20 & 33,9 \\
\hline & Sim & 20 & 64,5 & 19 & 67,9 & $\geq 0,05$ & 39 & 66,1 \\
\hline \multirow{2}{*}{$\begin{array}{c}\text { RT. } \\
\text { Axila } \\
\end{array}$} & Não & 29 & 93,5 & 25 & 89,3 & $=0,003$ & 54 & 1,5 \\
\hline & Sim & 2 & 6,5 & 3 & 10,7 & $\geq 0,05$ & 5 & 8,5 \\
\hline \multirow{2}{*}{ QT } & Não & 20 & 64,5 & 20 & 71,4 & $\geq 0,05$ & 40 & 7,8 \\
\hline & Sim & 11 & 35,5 & 8 & 28,6 & $\geq 0,05$ & 19 & 32,2 \\
\hline \multirow{2}{*}{ HT } & Não & 21 & 67,7 & 14 & 50 & $\geq 0,05$ & 35 & 59,3 \\
\hline & Sim & 10 & 32,3 & 14 & 50 & $\geq 0,05$ & 24 & 40,7 \\
\hline
\end{tabular}

* RT: Radioterapia, QT: Quimioterapia, HT: Hormonioterapia. Teste Qui-quadrado de Pearson. 
Quando analisado, a associação entre o tempo pós cirurgia e linfedema, pelo Teste Qui-quadrado de Pearson, pode-se observar que associação é significativa $(p=0,006)$, quando analisado de 6 a 24 meses e acima de 24 meses (Tabela 4).

Tabela 4. Associação entre o tempo pós-cirúrgico e o aparecimento do linfedema em 59 mulheres submetidas ao tratamento cirúrgico de neoplasia maligna mamária 2008-2009

\begin{tabular}{|c|c|c|c|c|c|c|c|}
\hline \multirow{3}{*}{ Tempo pós-cirúrgico } & \multicolumn{4}{|c|}{ Presença de Linfedema } & & \multirow{2}{*}{\multicolumn{2}{|c|}{ Total }} \\
\hline & \multicolumn{2}{|c|}{ Não } & \multicolumn{2}{|c|}{ Sim } & \multirow[b]{2}{*}{ Valor de $p$} & & \\
\hline & $\mathrm{N}$ & $\%$ & $N$ & $\%$ & & $\mathrm{~N}$ & $\%$ \\
\hline Menor que 6 meses & 17 & 56,7 & 5 & 17,9 & $\geq 0,05$ & 22 & 37,9 \\
\hline De 6 a 24 meses & 10 & 33,3 & 14 & 50 & $=0,003$ & 24 & 41,4 \\
\hline Mais que 24 meses & 3 & 10 & 9 & 32,1 & $=0,003$ & 12 & 20,7 \\
\hline Total & 30 & 100 & 28 & 100 & - & 58 & 100 \\
\hline
\end{tabular}

Teste Qui-quadrado de Pearson.

\section{Discussão}

O presente estudo vem corroborar com o que é descrito na literatura de quão prevalente é o linfedema pós-tratamento secundário da neoplasia maligna da mama e a expressiva associação a obesidade.-12 Revisões de literatura demonstram incidências de 6,7 a 62,5\% oscilando de acordo com as variáveis avaliadas 9,13 , assim como do critério diagnóstico empregado. 12

O linfedema tem caráter multifatorial, tendo fatores relacionados à cirurgia, ao paciente e à doença propriamente dita..14,15 Dentre os fatores relacionados à cirurgia, a extensão da dissecção axilar parece ser o fator de risco mais importante na etiologia do edema de membro superior..$^{15}$

Além disso, quando associado à radioterapia axilar, a literatura relata aumento significativo de linfedema., ${ }^{2,13}$ Todavia, nesse presente estudo, não encontramos presença significativa de linfedema nas mulheres que foram submetidas a radioterapia axilar, trazendo uma discussão que outras variáveis podem ser mais relevantes no desenvolvimento dessa morbidade, tais como a presença de presença de infecções pós-operatórias.

As técnicas de abordagem axilar como linfonodo sentinela (LS) parecem diminuir o risco desta morbidade16, desde que realizada cuidadosamente ${ }^{10}$, pois apresentam resultados promissores no estadiamento sem a necessidade de esvaziamentos ganglionares desnecessários como antigamente. ${ }^{17}$ Neste estudo, a maioria foi submetida a linfadenectomia axilar e uma porcentagem menor ao linfonodo sentinela; entretanto, a presença de linfedema nessa amostra não apresentou relação com essa variável, porém demonstrou associação do linfedema com a extensão do esvaziamento, bem como comprometimento axilar.

Quanto aos fatores relacionados à doença, sabe-se que estadiamento avançado da doença tem relação íntima o risco de linfedema, devido as abordagens cirúrgicas mais invasivas e comprometimento linfonodal14,18, sendo o diagnóstico precoce essencial para sua prevenção. Pudemos observar nesse estudo que apesar de não apresentar significância estatística, existe um aumento na presença de linfedema nos estadiamentos mais avançados, representado por 34 mulheres que estavam no estadiamento II e III, 20 apresentaram a presença de linfedema. Além disso, apresentaram presença de infecções, tais como erisipelas e radiodermites, um dos potencializadores no desenvolvimento do linfedema.

Todavia, entre os diversos fatores implicados na ocorrência do linfedema, idade e obesidade ${ }^{19-21}$ precisam ser ressaltados, ou seja, os nossos resultados demonstraram incidência de $47,5 \%$ de linfedema, sendo a idade média apresentada de 60,41 anos e 71\% apresentarem sobrepeso ou obesidade. É sabido que a idade superior a 45 $\operatorname{anos}^{19}$ se mostrou significativa na formação de linfedema nas mastectomias radicais modificadas. A idade é citada em diversos artigos como fator de risco relacionado com o linfedema ${ }^{10,15,21}$, apesar de controvérsias. 
A obesidade tem sido motivo de estudos relacionados ao edema de membro superior pós-cirúrgico 22,23 , pacientes submetidas à quandrantectomia apresentam a obesidade como o principal fator preditivo. ${ }^{14}$ Entretanto, a infecção dos membros superiores e o ganho de peso pós cirurgia foram os únicos fatores de risco com relações significativas para o desenvolvimento dessa intercorrência..$^{11} \mathrm{Em}$ nossa pesquisa encontramos associação de risco do desenvolvimento do linfedema e obesidade, quanto maior o IMC, maior a chance de seu aparecimento. O ganho de peso já traz consigo diversas alterações circulatórias que acabam por descompensar um sistema linfático já comprometido pela cirurgia, levando a uma facilitação no desenvolvimento dessa condição. $,,, 7,10$

Todavia, ensaios clínicos randomizados demonstram que a diminuição do peso por meio de dietas é promissora e que independentemente do tipo de dieta a perda ponderal parece ser método efetivo na redução do volume do braço durante o tratamento. ${ }^{20}$ Por ser morbidade crônica, multifatorial e incurável, deve haver maior enfoque na prevenção. Atualmente o controle do peso consiste medida preventiva na maioria dos Guidelines ${ }^{9}$, sendo apenas uma recomendação das sociedades em consensos. ${ }^{1.5}$

Apesar das complicações, a cirurgia e radioterapia são essenciais no manejo e tratamento da maioria das pacientes com câncer de mama, sendo que os benefícios ultrapassam o risco de linfedema. Desta maneira devemos atuar nos agentes causais preveníveis como a obesidade, sem subestimar a incapacidade funcional, a severidade dos sintomas, aflições psicológicas e morbidade das pacientes, sendo deplorável a conduta niilista. 10

Ademais, o linfedema se tornará mais prevalente na medida em que a sobrevida aumentar 13 , sendo a morbidade mais estressante. ${ }^{9}$ Às vezes o linfedema tem um impacto muito maior na piora da qualidade de vida do que a própria cirurgia, pois a última pode ser mais facilmente disfarçada em detrimento ao edema..$^{10}$ Alguns sintomas subjetivos também são associados significativamente com o linfedema entre eles a parestesia ${ }^{18}$, porém métodos exclusivamente qualitativos não demonstram com segurança o impacto funcional do linfedema ${ }^{12}$, porém dados como esses não foram devidamente identificados nesse estudo.
Do mesmo modo medidas profiláticas para redução de sua ocorrência, intervenções fisioterapêuticas no decorrer do pós-operatório, tal qual a recomendação da redução de peso deve ser estimulada, principalmente quando implicam diretamente na qualidade de vida das pacientes. Entretanto, algumas limitações do estudo podem ser ressaltadas, como a dificuldade em estabelecer fatores risco exatos para o desenvolvimento do linfedema e a investigação da atuação da Fisioterapia na prevenção e/ou tratamento dessa condição nesse grupo. Os autores sugerem estudos prospectivos, longitudinal nos centros especializados em câncer de mama, com a proposta de investigar estas variáveis no linfedema.

\section{Conclusão}

Existe correlação entre obesidade e desenvolvimento do linfedema. Os tipos de tratamento parecem não ter influenciado o seu desenvolvimento e quanto mais tempo decorrido pós- cirurgia, maior o risco do seu aparecimento.

\section{Contribuições dos autores}

Antonini M participou da coleta de dados, construção dos resultados e revisão final do artigo. Salerno GRF participou da construção dos resultados e revisão final do artigo. Moro RS participou da coleta de dados e análise dos resultados. Oliveira MVO e Ferraro O participaram da coleta de dados e revisão da escrita. Lopes RG participou da análise e construção dos resultados e revisão final do artigo científico.

\section{Conflitos de interesses}

Nenhum conflito financeiro, legal ou político envolvendo terceiros (governo, empresas e fundações privadas, etc.) foi declarado para nenhum aspecto do trabalho submetido (incluindo, mas não se limitando a subvenções e financiamentos, participação em conselho consultivo, desenho de estudo, preparação de manuscrito, análise estatística, etc.).

\section{Referências}

1. Instituto Nacional de Câncer. Estatísticas do câncer [Internet]. Brasil: Ministério da Saúde; 2021. Disponível em: https://www. inca.gov.br/numeros-de-cancer 
2. Carli AC. Diagnóstico por imagem em doenças da mama. In: Boff RA, Wisintainet F. Mastologia moderna: abordagem multidisciplinar. Caxias do Sul: Mesa Redonda; 2006.

3. Rezende LF, Rocha AVR, Gomes CS. Avaliação dos fatores de risco no linfedema pós-tratamento de câncer de mama. J Vasc Bras. 2010;9(4):233-38. http://dx.doi.org/10.1590/S167754492010000400005

4. Tacani PM, Camargo RAL, Silva G, Moreira BC, Batista PAN, Montezello D, et al. Fisioterapia descongestiva no linfedema de membros superiores pós-mastectomia: estudo retrospectomia. Rev Bras Ciên Saúde. 2013;37(11):17-23. https://doi.org/10.13037/ rbcs.vol11n37.1884

5. Executive Committee. The diagnosis and treatment of peripheral lymphedema: 2016 Consensus document of the international society of Lymphology. Lymphology. 2016;49(4):17084. Citado em: PMID: 29908550

6. Elias S, Facina G, Araujo Neto JT, organizadores. Mastologia. Condutas atuais. São Paulo: Manole; 2016.

7. Durant LC, Tomadon A, Camboin FF, Silva J, Campos RB, Gozzo TO. Sobrevivência e Fatores de Risco em Mulheres com Câncer de Mama: a Relação do Linfedema. Rev. Bras. Cancerol. 2019;65(1):e07303. https://doi.org/10.32635/2176-9745.RBC.2019v65n1.303

8. Fretta TB, Boing L, Bussmann RM, Guimarães AC. Pain rehabilitation treatment for women with breast cancer. BrJP. 2019;2(3):279-83. https://doi.org/10.5935/2595-0118.20190049

9. National Comprehensive Cancer Network. NCCN Guidelines Version 5.2020. Breast cancer [Internet]. Disponível em: https:// www2.tri-kobe.org/nccn/guideline/breast/english/breast.pdf

10. Paiva CB, Dutra CMS. Prevalência de linfedema após tratamento de cancer de mama em pacientes com sobrepeso. Fisioter Pesqui. 2016;23(3):263-7. https://doi.org/10.1590/18092950/15214123032016

11. Ribeiro RVE. Prevalência de linfedema após mastectomia em portadoras de câncer de mama: uma Revisão sistemática acerca da influência da reconstrução imediata. Rev. Bras. Cir. Plást. 2019;34(1):113-9. http://dx.doi.org/10.5935/21771235.2019RBCP0017

12. Wolfs J, Beugels J, Kimman M, Grzymala AAP, Heuts E, Keuter $X$, et al. Improving the quality of life of patients with breast cancer-related lymphoedema by lymphaticovenous anastomosis (LVA): study protocol of a multicentre randomised controlled trial. BMJ Open. 2020;10:e035337. http://dx.doi.org/10.1136/ bmjopen-2019-035337

13. Bergmann AM, Echenique I, Koifman RJ. Incidência e prevalência de linfedema após tratamento cirúrgico do câncer de mama: revisão da literatura. Rev Bras Cancerol [Internet]. 2007;53(4):461-70. Disponível em: https://rbc.inca.gov.br/site/ arquivos/n_53/v04/pdf/revisao3.pdf
14. Goel A, Agarwal J, Mehta S, Kumar K. Arm lymphedema after treatment of breast cancer: Etiology, diagnosis, and management. Asian Journal of Oncology. 2015;1(2):77-86. http://dx.doi. org/10.4103/2454-6798.173284

15. Harris JR, Lippman ME, Morrow M, Osborne CK. Diseases of the breast. $5^{\mathrm{a}}$ ed. Philadelphia: Wolters Kluwer; 2014.

16. Quadros LGA, Gebrim LH. A pesquisa do linfonodo sentinela para o câncer de mama na prática clínica do ginecologista brasileiro. Rev Bras Ginecol Obstet. 2007;29(3):158-64. https://doi. org/10.1590/S0100-72032007000300008

17. Magaldi CM, Barros ACSD, Magaldi FM, Mantese JC, Pinotti JA. Avaliação da morbidade e funcionalidade do membro superior e mulheres submetidas à linfedenectomia axilar total e biopsia de linfonodo sentinela por câncer de mama. Rev Bras Mastologia [Internet]. 2005;15(1):9-14. Disponível em: https://pesquisa. bvsalud.org/ripsa/resource/pt/lil-424774

18. Gozzo TO, Aguado G, Tomadon A, Panobianco MS, Prado MAS. Perfil de mulheres com linfedema no pós-tratamento de câncer de mama. Esc Anna Nery. 2019;23(4):e20190090. https://doi. org/10.1590/2177-9465-ean-2019-0090

19. Wanchai RNA, Armer JM CLT, Stewart R, Lasinski BB. Breast Cancer-Related Lymphedema: A Literature Review for Clinical Practice. Int. J. Nurs. Sci. 2016;3(2):202-7. https://doi.org/10.1016/j. ijnss.2016.04.006

20. Tsai CL, Hsu CY, Chang WW, Lin YN. Effects of weight reduction on the breast cancer-related lymphedema: A systematic review and meta-analysis. The Breast. 2020:52:116-121. https://doi. org/10.1016/j.breast.2020.05.007

21. Bojinović-Rodić D, Popović-Petrović S, Tomić S, Markez S, Živanić D. Upper extremity function and quality of life in patients with breast cancer related lymphedema. Vojnosanit Pregl. 2016;73(9):825-30. https://doi.org/10.2298/vsp150208075b

22. Avelar AA, Derchain SFM, Camargo CPP, Lourenço LS, Sarian LOZ, Yoshida A. Qualidade de vida, ansiedade e depressão em mulheres com câncer de mama antes e após a cirurgia. Rev. Ciênc. Méd [Internet]. 2006;15(1):1120. Disponível em: http:// seer.sis.puc-campinas.edu.br/seer/index.php/cienciasmedicas/ article/viewFile/1131/1106

23. Rosa JI, Salomé GM, Miranda FD. Construção e validação de um algoritmo prevenção e tratamento de linfedema de membros superiores. Fisioter. Mov. 2020;33: e003367. https://doi. org/10.1590/1980-5918.032.ao66 